\title{
SCHEDULING METHOD IN DESIGN OF MULTIPURPOSE BATCH PLANTS WITH CONSTRAINED RESOURCES
}

\author{
TETSUO FUCHINO* AND MASAAKI MURAKI \\ Department of Industrial Engineering and Management, \\ Tokyo Institute of Technology, Tokyo, 152 \\ TOYOHIKO HAYAKAWA \\ Department of Industrial Engineering, Chiba Institute of Technology, \\ Narashino, 275
}

Key Words: Systems Engineering, Scheduling, Constrained Resources, On-site Work, Cyclic Production, Cyclic Scheduling, Multipurpose Batch Plants, Integer Programing Model, Design

\begin{abstract}
A scheduling method that incorporates the design of multipurpose batch plants is developed. The scheduling consists of two interactive sub-problems: production scheduling and constrained resources scheduling, and on-site work is one of the typical constrained resources. In the design of these plants, the optimal schedule should be made within a short time, and cyclic scheduling according to the cycle production is appropriate. An IP (Integer Programing) model is suitable for such short-term scheduling, and the scheduling method is developed on the basis of the previous IP model for production scheduling. To make the optimal schedule, the allocation and sequence of tasks of on-site work should be optimized in relation with the production scheduling. In this study, the required properties of the on-site work scheduling in design is considered, and this sub-problem is converted into an assignment problem. The on-site work is defined in linear constraints with integer variables describing the task assignments. Moreover, the on-site work is newly defined in the production scheduling, and these definitions are related by the introduced integer variables. Finally, the effectiveness of the developed scheduling method is demonstrated by an example problem.
\end{abstract}

\section{Introduction}

Multipurpose batch plants, which are suitable for producing a variety of high-value added products in low volume, have become of interest in recent years. Only well-designed plants can produce such products effectively, and the design problem is one of minimizing the total equipment costunder the constraints of the production requirements and the recipe data for each product. However, the decision variables for this problem, the plant configuration and equipment sizes, cannot be optimized without scheduling ${ }^{3)}$. Thus scheduling must be incorporated in the design.

In designing batch plants, the processing paths for each product are given for a plant configuration, and the purpose of scheduling is maximizing the utilization of the equipment in this plant configuration so as to optimize the equipment size. The optimal plant configuration is searched while optimizing the equipment sizes through scheduling, so that the schedule is necessarily made within a short time in design. To maximize the utilization of equipment for a given plant configuration, the interactions between products should be considered. Cyclic scheduling, which is based on cyclic production, is appropriate ${ }^{1)}$ forconsidering theseinteractions within a short time, because the cycle time for only one cycle

\footnotetext{
* Received November 4, 1993. Correspondence concerning this article should be addressed to T. Fuchino.
}

should be minimized. However, the scheduling problem consists of two interactive sub-problems: production scheduling and constrainedresourcescheduling. In multipurpose batch plants, products are made by respective processing paths through the plants ${ }^{5)}$, and the operation sequences in each equipment unit should be optimized in the production scheduling. On the other hand, batch plants need a number of resources such as process utilities, intermediate storage, on-site work and so on with certain constraints, so that the usage of these resources should be considered at the design stage in constrained resources scheduling. The operation sequences in production scheduling and the usage of resources are interactive with each other, so that the above two kinds of schedules must be made simultaneously.

There are various scheduling methods for batch plants in the literature, but most of them focus attention only on the production scheduling. Moreover, these methods for production scheduling ${ }^{1,6)}$ are for multiproductbatch plants, in which the productsare produced with essentially the same processing paths ${ }^{5)}$. Thus only the production order is optimized, and these methods cannot be applied to multipurpose batch plants. Fuchino et al. ${ }^{2)}$ developed an integer programing (IP) model for the production schedule of multipurpose batch plants, which can optimize the operation sequences in each 


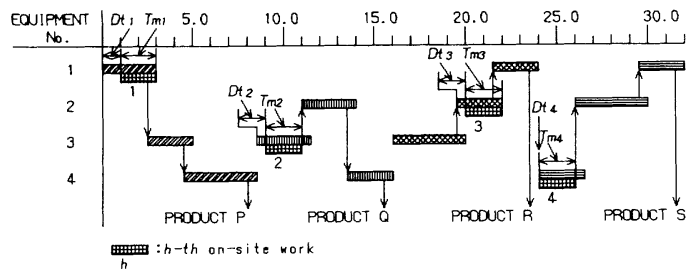

Fig. 1 Processing Paths for Products P, Q, R and S

equipmentunitsimultaneously. However, thismodel was not planned to consider constrained resources. On the other hand, a few studies address constrained resources. $\mathrm{Ku}$ and Karimi ${ }^{7}$ adoptedintermediate storage astheconstrained resource, and they proposed an algorithm for intermediate-storage scheduling by using dispatching rules for a given production schedule. Their algorithm is for multiproduct batch plants, so that the constrained resource schedule can be made for a given production schedule by adjusting the time of starting operation of the equipment. However, the operation sequences in each equipment unit are different in the multipurpose batch plants, so that such adjustments are usually infeasible in these plants (discussed in the next section in detail). Kondili et al. ${ }^{4)}$ and Shah et al ${ }^{8)}$ formulated the scheduling problem of multipurpose batch plants with constrained resources for the IPmodel by applying material balance calculations. However, their formulation requires an excessively large number of integer variables, even for cyclic scheduling. Moreover, the material balancecalculationcannotbeappliedforanyconstrained resources other than process utilities.

Among the constrained resources, on-site work is typical in batch plants. Many tasks of on-site work, such as compositional adjustments and casting small amount of solid materials, are needed in various equipment according to the product. These tasks are necessarily carried out by a finite number of site operators. To manage the on-site work, allocations of these tasks to the site operators and the sequences of the allocated tasks must be optimized. All usage of the resources has this feature, so that on-site work scheduling is not only practically important but is also applicable to any other constrained resources scheduling. Accordingly, the purpose of this study is to develop a cyclic scheduling method for the design of the multipurpose batch plants.

The scheduling is fundamentally a combinatorial optimization problem including continuous variables, and an IP model is appropriate for a short-term scheduling such as cyclic scheduling to satisfy the necessary optimality. Concerning the production scheduling, the concept of the IP model developed by Fuchino et al. ${ }^{2)}$ can be applied, and the scheduling method in design is considered on the basis of this IP model in this study.

The two sub-problems of scheduling, the production and the on-site work scheduling, are interactive with each other. The on-site work scheduling consists not only of the sequencing problem but also that of allocation. Thus it was difficult to formulate the on-site work sched-
Table 1. Processing Time

\begin{tabular}{ccccc}
\hline \multicolumn{5}{c}{ Equipment } \\
\hline Product & 1 & 2 & 3 & 4 \\
\hline$P$ & 2.0 & - & 1.5 & 3.0 \\
$Q$ & - & 2.0 & 2.0 & 1.5 \\
$R$ & 1.5 & 1.5 & 3.0 & - \\
$S$ & 2.0 & 3.0 & - & 1.5 \\
\hline
\end{tabular}

uling related to the production scheduling of the IPmodel suitably. In this study, the required property of onsite work scheduling in design is considered, and the onsite work scheduling is converted into an assignment problem. Integer variables to describe the task assignments are introduced instead of the allocation and sequence of tasks, and the time of starting the tasks is defined in the on-site work scheduling. The time of starting is newly defined in the production scheduling to consider theinteractions betweenthe production and the on-site work scheduling, and the relations between these definitions are linearized with integer variables.

In the following sections, the characteristic of scheduling for multipurpose batch plants is discussed, and a developed IP-model is presented. The effectiveness of this model is demonstrated by solving an example problem.

\section{Characteristics of Scheduling for Multipurpose Batch plants}

In this section, the characteristic of scheduling for multipurpose batch plants is considered with a simple example. Before designing, the kinds of products and number of batches for these products in one cycle are fixed as the production requirements, and the following items are specified in the recipe data

- Processing time for each operation

- Charging and/or discharging time

- Clean-up time for successive products

- Operations in which tasks of on-site work are needed

- Relative time of starting task in operation

- Necessary time for each task

- Stability of intermediate products

Moreover, the number of site operators is limited, and the processing paths for each product are given for a plant configuration. In this example, four products $(P, Q$, $\mathrm{R}$ and $\mathrm{S}$ ) are considered, and the number of batches for each product in one cycle is one here. The plant is composed of four equipment units, and the processing paths for each product are as shown in Fig. 1. The processing time for each operation is shown in Table 1, and the charging and/or discharging time is fixed at 0.5. The clean-up time is neglected for simplicity here. Four tasks of on-site work ( $h=1$ to 4 ) are needed, as shown in Fig. 1 , and these tasks are carried out by two site operators. The relative time difference between the time of starting the tasks and operations $\left(D t_{h}\right)$ and the necessary time for 
Table 2. Parameters for On-site Work Scheduling

\begin{tabular}{ccccc}
\hline & \multicolumn{5}{c}{ Task- $h$} \\
\hline$h$ & 1 & 2 & 3 & 4 \\
\hline$D t_{h}$ & 1.0 & 0.5 & 0.5 & 0.0 \\
$T m_{h}$ & 2.0 & 2.0 & 2.0 & 2.0 \\
\hline
\end{tabular}

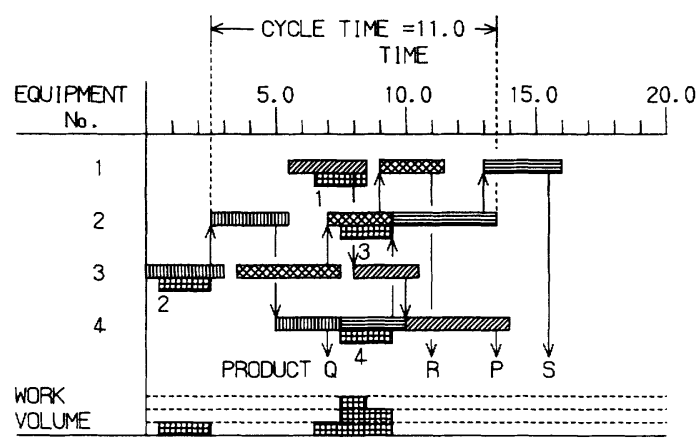

Fig. 2 Production Schedule for Fig. 1

these tasks $\left(T m_{h}\right)$ are shown in Table 2. The intermediate products are often unstable in chemical plants, so that the zero wait policy ${ }^{1)}$ is adopted.

When only the production schedule is made by the previous IP-model ${ }^{2)}$, then the one-cycle schedule shown in Fig. 2 is obtained. According to this schedule, the production order is Q-R-P-S, but the operation sequences in each equipment unit are not the same as this order because of the production manner of the multipurpose batch plants. For example, the operation sequences in the first and fourth equipment units are P-R-S and Q-S-P respectively. The cycle time is minimized to 11.0 . However, two site operators must carry out three tasks of onsite work (1,3 and 4) simultaneously, so that this schedule is infeasible.

In the previous study ${ }^{7)}$, the constrained resource schedule (on-site work schedule) was made for a given production schedule (Fig. 2) by adjusting the time of starting the operations. According to Fig. 2, tasks 1, 3 and 4 should be carried out simultaneously, so that the time of starting only one of these tasks should be changed to allocate them to two site operators. When task 4 is considered, for example, the operations for product $S$ should be shifted independently to change the time of starting only for task 4 . However, the operation sequences in each equipment unit are different from those in the other units. These sequences for products $P$, $R$ and $S$ in each equipment unit are P-R-S, R-S, R-P and $S-P$ respectively. If the operations for product $S$ is shifted, then the operations for products $P$ and $R$ are also moved in the same direction as product $S$. The same situation will occur for tasks 1 and 3. Thus the time of starting a task cannotbe adjusted independently, and the tasks of on-site work 1, 3 and 4 cannot be allocated to two site operators. This feature is caused by the nature of production in multipurpose batch plants.

On the other hand, when both the production

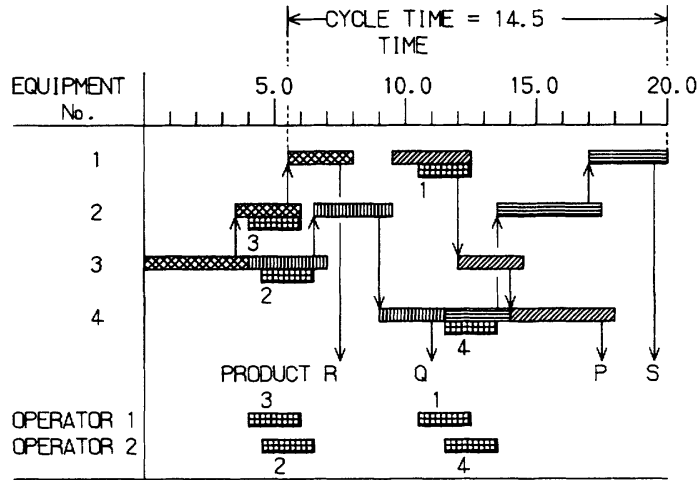

Fig. 3 Optimal Schedule for Fig. 1

scheduling and the on-site work scheduling are carried out simultaneously, then the one-cycle schedule as shown in Fig. 3 can be made. In this schedule, the operation sequences in each equipment are different from Fig. 2 , and the cycle time is extended from 11.0 to 14.5. However, tasks 3, 1 and 2, 4 are allocated respectively to the site operators 1 and 2 in these sequences as shown in Fig. 3, and a feasible and optimal schedule is obtained. If a multipurpose batch plant is designed unreasonably through the schedule as shown in Fig. 2, then the batch plantcannotsatisfytheproductionrequirements. Therefore, the production and on-site work schedule should be made simultaneously to design the optimal one-cycle schedule.

To make the production and on-site work schedule simultaneously, these two kinds of scheduling should be related. Moreover, the allocations of each task to the site operators and the sequences of the allocated tasks should be formulated into constraints for the on-site work scheduling. In this study, the tasks of on-site work are assumed to be allocated uniformly to the site operators from the consideration of the required property of the on-site work scheduling in design. Then the number of tasks to be carried out by each operator is specified beforehand, and the on-site work scheduling can be convertedintoanassignmentproblem. Theintegervariables to describe the assignments are introduced. Then the allocations and sequences of the tasks can be considered with these integer variables. Furthermore, the time of starting the tasks is defined respectively in the production and on-site work scheduling, and these definitions are related tothelinearizedconstraintsby theintroduced integer variables. Consequently, an IP-model for incorporating scheduling in the design of multipurpose batch plants is developed, and the developed IP-model is explained by using this example problem in the next section.

\section{IP-model}

The developed IP-model is explained by using the small example problem that is used in the previous section. Four products $(\mathrm{P}, \mathrm{Q}, \mathrm{R}$ and $\mathrm{S})$ are planned to be produced with respective processing paths through four 


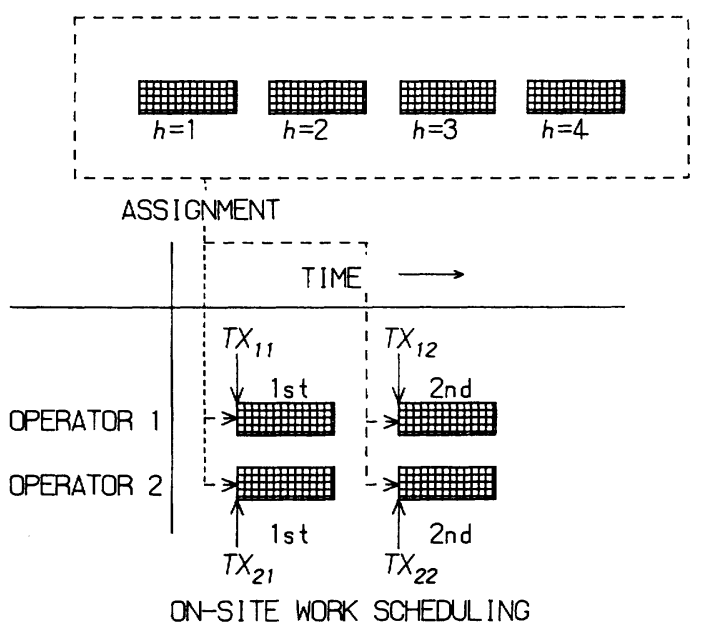

Fig. 4 Assignment Problem for On-site Work Scheduling

units of equipment, and four tasks of on-site work ( $h: h=$ 1 to 4) are carried out by two site operators as shown in Fig. 1. To describe the interaction between production and on-site work scheduling, the tasks of on-site work are defined with their time of starting respectively in these two kinds of scheduling, so that constraints to describe the following items are necessary.

- Definitions of tasks in on-site work scheduling.

- Definitions of tasks in production scheduling.

- Relations between these definitions.

Regarding the production scheduling, the concept of the IP-model developed by Fuchino et al. ${ }^{2)}$ can be applied, so that the formulation of the on-site work scheduling is explained for the first step. Then the tasks of on-site work can be defined with their time of starting from the viewpoint of site operators.

The on-site work scheduling in design is to restrict the number of tasks carried out at the same time for calculating the feasible and optimal cycle time, so that it is not necessary to distinguish the difference between site operators. Thus it can be assumed that each site operator carries out the same number of tasks. In this example, each site operator is assumed to carry out two tasks as shown in Fig.4, and allocating and sequencing problems for the on-site work scheduling can be converted into an assignment problem.

When $0 / 1$ integer variables $(M)$ satisfying the conditions as shown in Eqs. (1) and (2), and the time of starting each task from the viewpoint of site operators $(T X)$ as shown in Fig. 4, are introduced, this assignment problem can be described by the relation between the successive tasks shown in Eq. (3). Moreover, the cycle time $(T C)$ in the on-site work scheduling can be defined as shown in Eq. (4).

$$
\begin{gathered}
\sum_{f=1}^{E} \sum_{g=1}^{G_{f}} M_{f, g, h}=1.0(h=1 \text { to } H) \\
\sum_{h=1}^{H} M_{f, g, h}=1.0(f=1 \text { to } F)\left(g=1 \text { to } G_{f}\right)
\end{gathered}
$$

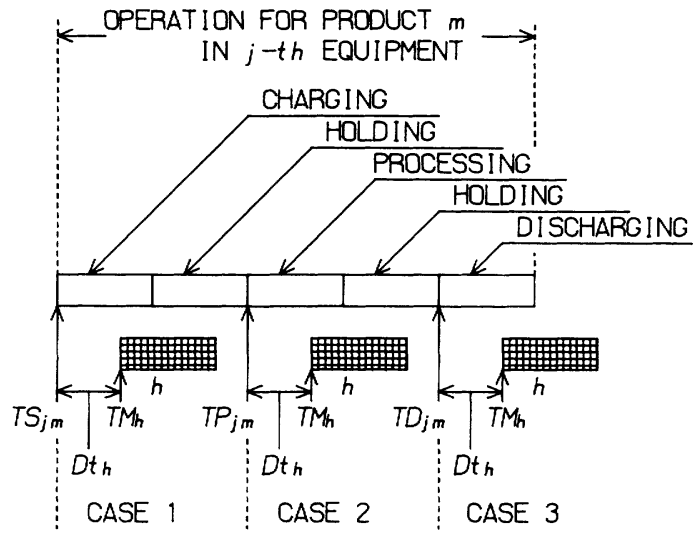

Fig. 5 Definition of Task within Operation

$$
\begin{gathered}
T X_{f, g+1}-T X_{f, g} \geq \sum_{h=1}^{H} T m_{h} \cdot M_{f, g, h}\left(g=1 \text { to } G_{f}\right) \\
T C \geq T X_{f, G_{f}+1}-T X_{f, 1} f=1 \text { to } F
\end{gathered}
$$

where $M_{f, g, h}$ is equal to one if and only if the site operator $f$ carries out task $h$ in the $g$-th sequence. $T X_{f, g}$ is the time of starting the $g$-th task of the site operator $f . T_{h}$ is the necessary time for task $h$, and it is specified in the recipe data from products and/or the type of $h$ as shown in Table 2. $F$ is the number of operators ( $F=2$ here), and $G_{f}$ is the number of tasks allocated to the operator $f\left(G_{1}=\right.$ 2 and $\left.G_{2}=2\right)$. $H$ is the total number of tasks $(H=4)$. Therefore, the on-site work scheduling can be expressed within linear constraints, and the tasks of on-site work are defined with their time of starting $\left(T X_{f, g}\right)$.

On the other hand, to consider the interaction between the production and the on-site work scheduling, tasks should be defined also in the production scheduling. In the production scheduling developed by Fuchino $e$ al. $^{2}$, each operation is divided into five suboperations: charging, holding, processing, holding and discharging. The time of starting the charging $\left(T S_{j, m}\right)$, the processing $\left(T P_{j, m}\right)$ and discharging $\left(T D_{j, m}\right)$ for the product $m$ in the equipment $j$ are introduced as variables as shown in Fig. 5. The on-site work is required as charging (case 1), processing (case 2) or discharging (case 3) depending on the type of task, so that the time of starting a task can be related to the time of starting these sub-operations as shown in Fig. 5. $D t_{h}$ is the relative time difference between the time of starting a task $h$ and a sub-operation, and it is specified in the recipe data from products and/or a type of $h$ as shown in Table 2 . When the time of starting the tasks from the viewpoint of operation $\left(T M_{h}\right)$ is newly introduced as variables as shown in Fig. 5, then on-site work can be defined in the production scheduling as shown in Eqs. (5).

$$
T M_{h}-T S_{m, j}\left(T P_{m, j} \text { or } T D_{m, j}\right)=D t_{h}
$$

In this example, the zero wait policy is adopted, so that all the tasks can be defined with the time of starting the charging as shown in Eqs. (6) to (9). 


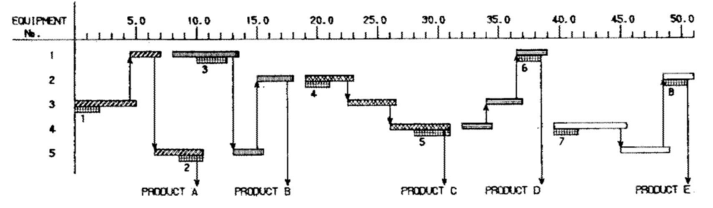

Fig. 6 Processing Paths for Products A, B, C, D and E

Table 3. Processing Time

\begin{tabular}{cccccc}
\hline \multicolumn{5}{c}{ Equipment } \\
\hline Product & 1 & 2 & 3 & 4 & 5 \\
\hline$A$ & 2.5 & - & 5.0 & - & 4.0 \\
$B$ & 5.5 & 3.0 & - & - & 2.5 \\
$C$ & - & 4.0 & 4.0 & 5.0 & - \\
$D$ & 2.5 & - & 3.0 & 2.5 & - \\
$E$ & - & 2.5 & - & 6.0 & 4.0 \\
\hline
\end{tabular}

Table 4. Clean-up Time

\begin{tabular}{cccccc}
\hline \multicolumn{6}{c}{ product to } \\
\hline product from & $A$ & $B$ & $C$ & $D$ & $E$ \\
\hline$A$ & - & 1.0 & 1.5 & 0.5 & 1.0 \\
$B$ & 1.5 & - & 1.0 & 1.0 & 1.5 \\
$C$ & 1.0 & 0.5 & - & 1.5 & 1.0 \\
$D$ & 0.5 & 1.5 & 0.5 & - & 1.5 \\
$E$ & 1.0 & 1.0 & 1.0 & 1.5 & - \\
\hline
\end{tabular}

$$
\begin{aligned}
& T M_{1}-T S_{P, 1}=D t_{1} \\
& T M_{2}-T S_{Q, 3}=D t_{2} \\
& T M_{3}-T S_{R, 2}=D t_{3} \\
& T M_{4}-T S_{S, 4}=D t_{4}
\end{aligned}
$$

In the first and second steps, the time of starting the tasks is defined respectively in production and on-site work scheduling with $T M_{h}$ and $T X_{f, g}$. Thus it is necessary to relate these definitions in the third step. The relations between $T M_{h}$ and $T X_{f, g}$ can be considered as shown in Eq. (10) separately.

$$
T X_{f, g}=\left\{\begin{array}{cl}
T M_{1} & \left(\text { if } M_{f, g, 1}=1\right) \\
T M_{2} & \left(\text { if } M_{f, g, 2}=1\right) \\
\vdots & \\
T M_{H} & \left(\text { if } M_{f, g, H}=1\right)
\end{array}(f=1 \text { to } F)\left(g=1 \text { to } G_{f}\right)\right.
$$

Eq. (10) can be rearranged into a nonlinear equation as Eq. (11) by adding each other after multiplying the continuous variables $T M_{h}$ by the introduced integer variables $M_{f, g, h}$.

$$
T X_{f, g}=\sum_{h=1}^{H}\left(M_{f, g, h} T M_{h}\right)(f=1 \text { to } F)\left(g=1 \text { to } G_{f}\right)
$$

This nonlinear constraint Eq. (11) can be linearized by setting up boundaries with the integer variables $M_{f, g, h}$ and a sufficiently large number $N$ as shown in Eq. (12).
Table 5. Parameters for On-site Work Scheduling

\begin{tabular}{ccccccccc}
\hline \multicolumn{8}{c}{ Task- $h$} \\
\hline$h$ & 1 & 2 & 3 & 4 & 5 & 6 & 7 & 8 \\
\hline$D t_{h}$ & 0.0 & 2.0 & 2.0 & 0.0 & 2.0 & 0.0 & 0.0 & 0.0 \\
$T m_{h}$ & 2.0 & 2.0 & 2.5 & 2.0 & 3.0 & 2.0 & 2.0 & 2.0 \\
\hline
\end{tabular}

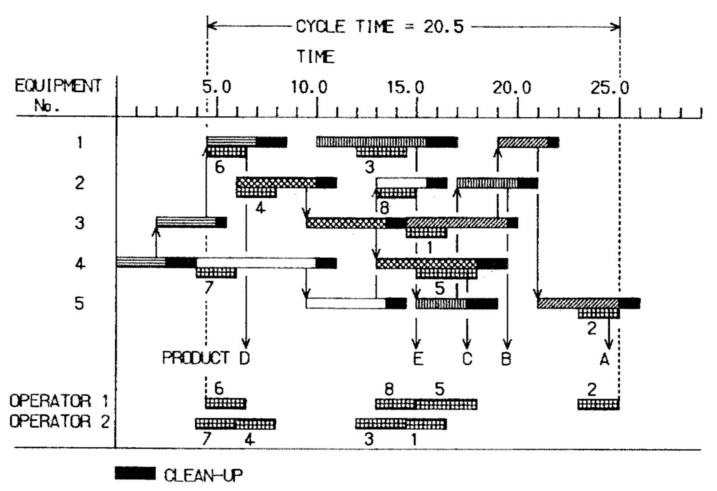

Fig. 7 Optimal Schedule for Fig. 6

$$
\begin{gathered}
N \cdot\left(M_{f, g, h}-1\right) \leq\left(T X_{f, g}-T M_{h}\right) \leq N \cdot\left(1-M_{f, g, h}\right) \\
(f=1 \text { to } F)\left(g=1 \text { to } G_{f}\right)
\end{gathered}
$$

Consequently, thenecessaryconstraintstodescribe the interaction between the production and on-site work scheduling canbeexpressed by linearcombinations with the integer variables, and the IP model for scheduling in design of multipurpose batch plants is developed. The IP-model can be optimized by iterative use of B.A.B. (Branch And Bound) and L.P. (Linear Programing) generally, and published software is available. In this study, the software AMPS by Fujitsu Ltd. on Sun-SPARCstation-IPX is used. The effectiveness of the developed model is demonstrated by solving an example problem in the next section.

\section{Example Problem}

In this section, an example scheduling problem of a multipurpose batch plant producing five products (A, B $\ldots \mathrm{E})$ is solved. The processing paths for these products are shown in Fig. 6, and eight tasks of on-site work ( $h=$ 1 to 8 ) are necessarily carried out by two site operators. The number of batches for these products in one cycle is one, and the zero wait policy is adopted. The recipe data for the production scheduling: processing time and clean-up time are shown in Tables 3 and 4 . The charging and discharging time is fixed at 0.5 here. The recipe data for on-site work scheduling: the necessary time for the tasks of on-site work $\left(\mathrm{Tm}_{h}\right)$ and the relative time differences between the time of starting the tasks and charging $\left(D t_{h}\right)$ are shown in Table 5.

The schedule obtained is shown in Fig. 7 as a Gantt chart. The cycle time for this example becomes 20.5 , and the sequences of the tasks are 6-8-5-2 and 7-4-3-1 respectively for each site operator. On the other hand, 


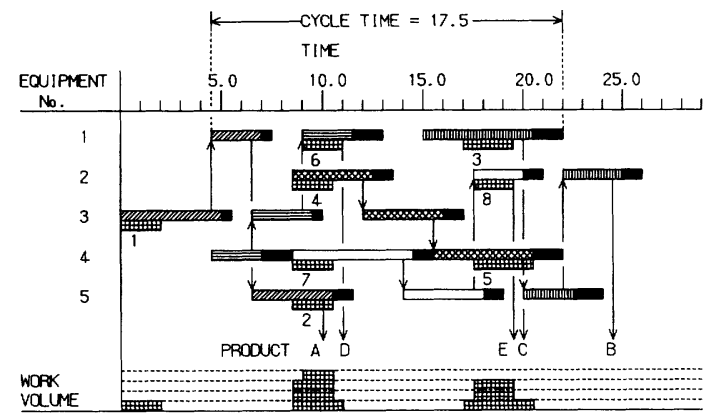

Fig. 8 Production Schedule for Fig. 6

when only the production schedule is made for this example, the schedule shown in Fig. 8 is obtained. The cycle time is 17.5 (20.5 in Fig. 7), but two site operators must carry out three and/or four tasks of on-site work simultaneously.

These results are summarized in Table 6. Clearly, whentheplantisdesignedunreasonablythroughproduction scheduling alone, the production requirements cannot be satisfied. Therefore, production and on-site work scheduling must be carried out simultaneously to make the optimal schedule in design of multipurpose batch plants, and the developed IP-model is appropriate.

\section{Conclusions}

A scheduling method that incorporates the design of multipurpose batch plants is developed. Scheduling consists of two interactive sub-problems: production scheduling and constrained resources scheduling, and on-site work is considered as the typical constrained resource in this study. In designing batch plants, the optimal schedule should be made within a short time, and cyclic scheduling, in which only one cycle is sufficiently optimized, is appropriate. An IP model is suitable for such short-term scheduling with the necessary optimality, and the scheduling method is developed on the basis of the previous IPmodel for production scheduling. However, the two sub-problems for scheduling are interactive with each other, and the on-site work scheduling consists of the allocating and sequencing of tasks.

In this study, the required property of on-site work scheduling in design is considered, and this sub-problem is converted into the assignment problem for the first step. Integer variables to describe the task assignments are introduced. The time of starting the tasks is defined by these integer variables, and on-site work scheduling is formulated into a practical size of the IP model. In the second step, the tasks of on-site work are newly defined in the production scheduling with the relative time of starting the tasks in each operation. In the first and second steps, the time of starting the tasks is defined independently, so that these definitions are related to the
Table 6. Summary

\begin{tabular}{cccc}
\hline & & Fig. 7 & Fig. 8 \\
\hline Cycle Time & & 20.5 & 17.5 \\
Production Sequence & & D-E-C-B-A & A-D-E-C-B \\
Operation Sequence & 1 & D-B-A & A-D-B \\
& 2 & C-E-B & C-E-B \\
& 3 & D-C-A & A-D-C \\
Task Sequence & 4 & D-E-C & D-E-C \\
& 5 & E-B-A & A-E-B \\
& Operator 1 & $6-8-5-2$ & Infeasible \\
& Operator 2 & $7-4-3-1$ & Infeasible \\
\hline
\end{tabular}

interactions between the on-site work scheduling and the production scheduling in the third step. These relations can be formulated into nonlinear equations, and these equations are linearized by setting up boundaries with theintroducedintegervariablesandthesufficientlylarge number. Finally, the effectiveness of the developed scheduling method is demonstrated by an example scheduling problem.

$\begin{array}{lll}\text { Nomenclature } & \\ D t & =\text { relative time difference } & {[-]} \\ F & =\text { number of site operators } & {[-]} \\ G & =\text { number of tasks } & {[-]} \\ H & =\text { total number of tasks } & {[-]} \\ M & =\text { integer variable } & {[-]} \\ N & =\text { sufficiently large number } & {[-]} \\ T C & =\text { cycle time } & {[-]} \\ T m & =\text { necessary time for task } & {[-]} \\ T M & =\text { time of starting task } & {[-]} \\ T D & =\text { time of starting discharging } & {[-]} \\ T P & =\text { time of starting processing } & {[-]} \\ T S & =\text { time of starting charging } & {[-]} \\ T X & =\text { time of starting task } & {[-]}\end{array}$

$<$ Subscripts $>$

$\begin{array}{ll}f & =\text { site operator } \\ g & =\text { sequence of on-site work } \\ h & =\text { on-site work } \\ j & =\text { equipment module } \\ m & =\text { product }\end{array}$

Literature Cited

1) Birewar, D.B. and I.E. Grossmann: Comput. Chem. Engng., 13, 141-161 (1989)

2) Fuchino, T., M. Muraki and T. Hayakawa: J. Chem. Eng. Japan, 25, 250-256 (1992)

3) Fuchino, T., K. Yamaguchi, M. Muraki and T. Hayakawa: $J$. Chem. Eng. Japan, 27, 57-64 (1994)

4) Kondili, E., C.C. Pantelides and R.W.H. Sargent: Comput.Chem. Engng., 17, 211-227 (1993)

5) Ku, H.M., D. Rajagopalan and I. Karimi: Chem. Eng. Prog., 83, 35-45 (1987)

6) Ku, H.M. and I. Karimi: Ind. Eng. Chem. Res., 27, 1840-1848 (1988)

7) Ku, H.M. and I. Karimi: Comput. Chem. Engng., 14, 49-69 (1990)

8) Shah, N., C.C. Pantelides and R.W.H. Sargent: Comput. Chem. Engng., 17, 229-244 (1993)

(Presented in part at the 57th Annual Meeting of the Society of Chemical Engineers, Japan at Sakai, March, 1992) 